\section{TRANSMISSION THROUGH AND WAVE GUIDANCE ON METAL PLATES PERFORATED BY PERIODIC ARRAYS OF THROUGH-HOLES OF SUBWAVELENGTH COAXIAL CROSS- SECTION}

\author{
Vitaliy Lomakin, ${ }^{1}$ Shuqing $\mathbf{L i}^{2}{ }^{2}$ and Eric Michielssen ${ }^{3}$ \\ ${ }^{1}$ Department of Electrical and Computer Engineering, University of \\ California, San Diego, La Jolla, CA 92093 \\ 2 Department of Electrical and Computer Engineering, University of \\ Illinois at Urbana Champaign, Urbana, IL 61801 \\ ${ }^{3}$ Department of Electrical Engineering and Computer Science, \\ University of Michigan, Ann Arbor, Ml 48109
}

\section{Received 7 December 2006}

ABSTRACT: Perfect electrically conducting (PEC) plates perforated by doubly periodic arrays of through-holes of subwavelength coaxial cross-section permit enhanced transmission of plane waves and support surface wave guidance. The transmission enhancement is attributed to coupling of free-space fields to TEM and TE ${ }_{11}$ modal fields in holes and occurs via two distinct mechanisms. The first couples incident fields to local resonances (cavity modes) supported by individual holes. The second couples scattered fields (diffraction modes) to global resonances (surface waves). The surface waves arise due to strong interactions between the holes and can have a large propagation wavenumber. The presented PEC model is adequate in the microwave and terahertz regimes and insights into related wave phenomena at optical frequencies. (C) 2007 Wiley Periodicals, Inc. Microwave Opt Technol Lett 49: 1554-1558, 2007; Published online in Wiley InterScience (www.interscience.wiley.com). DOI 10.1002/mop.22484

Key words: transmission; periodic grating; annular holes; coaxial holes; surface waves

\section{INTRODUCTION}

Phenomena of enhanced transmission of electromagnetic fields through plates perforated by doubly periodic arrays of throughholes of subwavelength cross-section [1-12] bode to enable numerous scientific and engineering technologies involving nearfield microscopy, optical data storage, and microwave and optical filtering and switching [13-16]. These phenomena, which manifest themselves as strong peaks in the frequency/angular dependencies of transmission coefficient magnitudes, have been observed in the optical, terahertz, and microwave regimes for holes of simply- and multiply-connected cross-sections [1-12].

For simply-connected cross-sections the enhancement is known to arise from hole tunneling enabled by the interaction of scattered field diffraction modes and surface waves (SWs) supported by the structure [2-4]. For multiply-connected cross-sections there appears to be less of a consensus on the nature of the enhancement mechanism [8-12]. Early works of Baida and Van Labeke [8, 9] suggest that TEM modes play a key role in transmission enhancement through coaxial apertures under both normal and oblique incidence. Later works by these and other authors [10-12] as well as an old work by Roberts and McPhedran [7] attribute the transmission enhancement to the excitation of propagating $\mathrm{TE}_{11}$ modal fields in the coaxial through-holes while prescribing no role to the TEM modes. In these studies the $\mathrm{TE}_{11}$ mode was propagating because of the cutoff frequency reduction due to the effects introduced by the finite conductivity in the optical/visible regime or/and because the holes' size was chosen to be only marginally subwavelength. However, in a number of applications, e.g., near field probes, one can be interested in holes of size that is much smaller than the wavelength in the frequency regimes ranging from near infrared, where the finite conductivity plays an important role to terahertz and microwave, where metals can be safely modeled as electric conductors.

This letter studies phenomena of scattering from, and wave guidance on, perfect electrically conducting (PEC) plates perforated by arrays of through-holes of subwavelength coaxial crosssection that support no higher-order propagating modes. The letter shows that in this regime the perforated plates support phenomena of enhanced transmission of plane waves as well as SW guidance. The transmission enhancement has two separate origins. First, incident waves may couple to TEM modal fields that locally resonate in the through-holes. Second, higher-order scattered field diffraction modes may couple to SWs. The SWs are global resonances appearing due to interactions between through-holes mainly mediated by their TEM and $\mathrm{TE}_{11}$ aperture fields. The SWs can have a large propagation wavenumber, which can be important in applications. The phenomena described here to some extent mirror those occurring on plates loaded by periodic arrays of infinite slits [17-19]; the principal difference between the two lies in the complicated angular dependence of the present structure's scattering properties, which stems from the radial symmetry of the coaxial cross-section TEM modal field.

\section{STRUCTURE CONFIGURATION}

Consider a free-standing PEC plate that is perforated by a doubly periodic Cartesian array of through-holes of subwavelength coaxial cross-section. The plate occupies the space $-d / 2<z$ $<d / 2$, the array periodicities along the $x$ - and $y$-directions are $L_{x}$ and $L_{y}$, and the coaxial cross sections' inner and outer conductor radii are $r_{1}$ and $r_{2}$. The plate is excited by a $\mathrm{TM}_{z}$ plane wave of frequency $f$ with electric field normal to the $y$-axis that is incident from an angle $\theta$ with respect to the $z$-axis. In what follows, the time dependence $e^{j 2 \pi f t}$ is assumed and suppressed. Also, the hole crosssection is assumed to be small compared to the wavelength $\lambda$, i.e. $r_{1}<r_{2}<\lambda$ with $\lambda=c / f$ and $c$ the free-space wave speed. Due to this condition, only the TEM mode in the holes is propagating whereas all higher order modes are evanescent.

\section{WAVE PHENOMENA SUPPORTED BY DENSE ARRAYS OF COAXIAL HOLES}

This section elucidates wave phenomena supported by the structure in Figure 1 assuming the arrays are dense, i.e., characterized by subwavelength periodicities. Section 3.1 presents an approximate model for the transmission coefficient. Section 3.2 details phenomena of enhanced transmission associated with localized resonances supported by coaxial holes.

\subsection{Model of the Transmission Coefficient}

First, assume oblique incidence, i.e. $\theta \neq 0$, and consider a dense array of coaxial through holes with $2 r_{2}<\left\{L_{x}, L_{y}\right\}<\lambda$ (this restriction will be lifted later). In this regime, the scattered field is
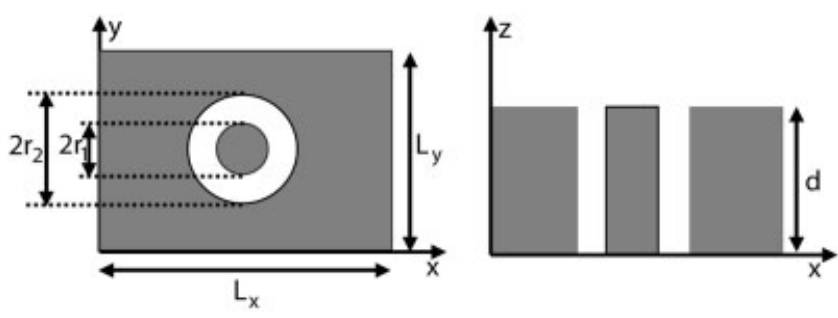

Figure 1 Structure configuration 
dominated by the zeroth-order diffraction mode outside the plate, and the TEM and $\mathrm{TE}_{11}$ modes in the coaxial through-holes. An approximate expression for the zeroth-order electric field transmission coefficient $T$ can be obtained via a standard modal approach [7-20]. For example, following Chen [20] and enforcing field continuity across the hole to free-space apertures while representing fields in terms of constituents with odd and even tangential electric field symmetry about the $z=0$ plane results in an integral equation in Ref. 20 [Eq. (1)] (see also Refs. 2 and 7), where the circular cross-section modes are replaced by coaxial cross-section ones. Projecting this equation onto, i.e. integrating over the unit cell with, a set of diffraction modes results in an infinite system of matrix equations for the excitation coefficients of the waveguide modes.

Next, it is noted that all higher (than zeroth) order diffraction modes cannot be excited strongly due to the fact that the periodicities $L_{x}$ and $L_{y}$ are assumed to be deeply subwavelength at this stage of consideration. Therefore only the dominant zeroth-order diffraction mode outside the plate can be taken into account in the infinite system of equations. Furthermore, the excitation of the higher (than TEM) order waveguide modes reduces as the mode cutoff frequency increases. To represent properly the influence of both TEM and cutoff modes while still keeping the physics simple and transparent, only the coefficient corresponding to the TEM and $\mathrm{TE}_{11}$ modes are kept in the above system of equations. Thus performed truncation of the infinite system of equations yields the following approximate expression for the transmission coefficient $T$

$$
\begin{aligned}
T & \approx \frac{\left|P_{00}\right|^{2} \tan \left(k_{0} d / 2\right)+\left|P_{01}\right|^{2} \tanh \left(\left|\zeta_{11}^{\mathrm{TE}}\right| k_{0} d / 2\right) /\left|\zeta_{11}^{\mathrm{TE}}\right|}{\cos \theta+j\left(\left|P_{00}\right|^{2} \tan \left(k_{0} d / 2\right)+\left|P_{01}\right|^{2} \tanh \left(\left|\zeta_{11}^{\mathrm{TE}}\right| k_{0} d / 2\right) /\left|\zeta_{11}^{\mathrm{TE}}\right|\right)} \\
& -\frac{\left|P_{00}\right|^{2} \cot \left(k_{0} d / 2\right)+\left|P_{01}\right|^{2} \operatorname{coth}\left(\left|\zeta_{11}^{\mathrm{TE}}\right| k_{0} d / 2\right) /\left|\zeta_{11}^{\mathrm{TE}}\right|}{\cos \theta+j\left(-\left|P_{00}\right|^{2} \cot \left(k_{0} d / 2\right)+\left|P_{01}\right|^{2} \operatorname{coth}\left(\left|\zeta_{11}^{\mathrm{TE}}\right| k_{0} d / 2\right) /\left|\zeta_{11}^{\mathrm{TE}}\right|\right)}
\end{aligned}
$$

The first and second terms are due to fields with odd and even tangential electric symmetry about the $z=0$ plane, respectively; $k_{0}=2 \pi f / c$ is the free-space wavenumber; $\zeta_{11}^{\mathrm{TE}} \approx(1$ $\left.-4 /\left(k_{0}\left(r_{1}+r_{2}\right)\right)^{2}\right)^{1 / 2}$ is the normalized wavenumber of the $\mathrm{TE}_{11}$ mode along the $z$-direction; and $P_{00}$ and $P_{01}$ are the coupling integrals of the tangential electric fields of the zeroth-order TM diffraction mode and the TEM and $\mathrm{TE}_{11}$ modes, respectively. Since the holes are small, $\left|P_{00}\right|,\left|P_{01}\right|<1$. Closed form expressions for $P_{00}$ and $P_{01}$ are not given as they are lengthy and do not provide additional physical insight.

Examination of the denominators of the terms on the right hand side in Eq. (1) reveals that $T$ has complex frequency or angle/ wavenumber poles, which are characteristic frequencies or angles/ wavenumbers of source-free fields supported by the perforated plate. The incident plane wave couples to these resonances when the frequency or angle of illumination is scanned through the real part of the poles. As a result, $|T|$ exhibits resonant peaks with bandwidth proportional to the imaginary part of the poles. Therefore, understanding the properties of these poles is essential to understanding the structure's transmission response. Two types of source-free fields may exist depending on whether $\sin \theta \lessgtr 1$.

\subsection{Transmission Phenomena}

When $\sin \theta<1$, the zeroth-order diffraction mode is a propagating plane wave and $\cos \theta=|\cos \theta|$. Recalling that $\left|P_{00}\right|,\left|P_{01}\right|<1$, the denominators in Eq. (1) have complex frequency solutions

$$
f_{p}^{\mathrm{loc}}=f_{r, p}^{\mathrm{loc}}+\delta_{p}^{\mathrm{loc}}
$$

where $p=o$ and $p=e$ refers to zeros of the denominator of the odd (first) and even (second) components in (1). The frequencies $f_{r, o}^{\text {loc }}=c(2 q-1) /(2 d)$ and $f_{r, e}^{\text {loc }}=c q / d$ with integer $q \geq 1$ approach $f_{o}^{\text {loc }}$ and $f_{e}^{\text {loc }}$, respectively; the $\delta_{p}^{\text {loc }}$ are (small) complexvalued displacements. The frequencies $f_{r, p}^{\text {loc }}$ characterize local resonances supported by individual through-hole cavities. These resonances are largely unaffected by cavity interactions; as a result, the $f_{p}^{\text {loc }}$ only weakly depend on the angle of incidence and array periodicities. Due to the presence of these poles, $|T|$ exhibits single transmission peaks as real $f$ scans through $\operatorname{Re}\left\{f_{p}^{\text {loc }}\right\}$. The peaks exhibit periodic behavior with respect to $d$ and have large magnitudes even for very thick plates.

To illustrate the transmission enhancement due to the local resonances, consider a perforated plate with structure parameters defined in the caption to Figure 2. All numerical results are obtained via a full wave modal analysis [19]. Figure 2(a) depicts

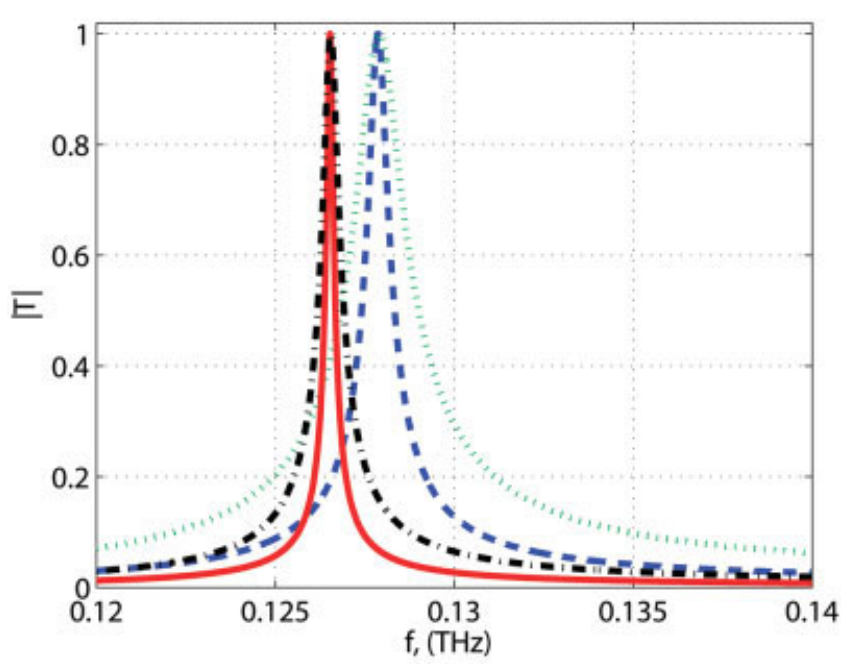

(a)

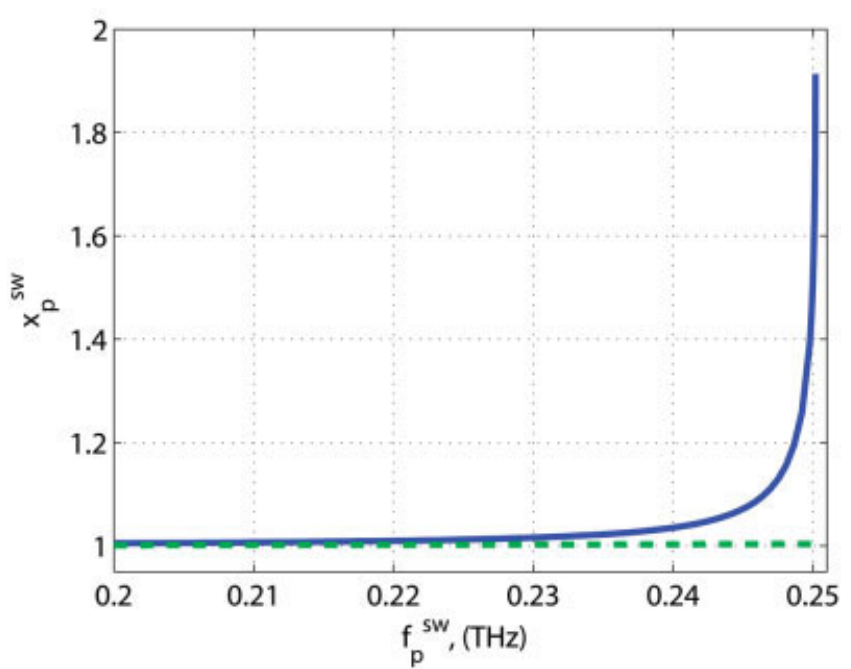

(b)

Figure 2 (a) Transmission coefficient $|T(f)|$ for $\sin \theta=0.5, L_{x}$ $=0.8 \mathrm{~mm}$ (solid line), $\sin \theta=0.5, L_{x}=0.31 \mathrm{~mm}$ (dashed line), $\sin \theta=0.7, L_{x}=0.31 \mathrm{~mm}$ (dotted line), and $\sin \theta=0.7, L_{x}$ $=0.8 \mathrm{~mm}$ (dashed dotted line); (b) Dispersion curves of $f_{e}^{\mathrm{sw}}\left(\zeta_{e}^{\mathrm{sw}}\right)$ (solid line) and $f_{o}^{\mathrm{sw}}\left(\zeta_{o}^{\mathrm{sw}}\right)$ (dashed line) for $L_{x}=0.31 \mathrm{~mm}$. Other structure parameters are $L_{y}=0.31 \mathrm{~mm}, r_{1}=0.075 \mathrm{~mm}, r_{2}=0.15 \mathrm{~mm}$, and $d$ $=1.1 \mathrm{~mm}$. [Color figure can be viewed in the online issue, which is available at www.interscience.wiley.com] 
the dependence $|T(f)|$ near a local odd resonance associated with $f_{o}^{\text {doc }}$ and $f_{r, o}^{\text {doc }}=c /(2 d)$ for different periodicities and angles of incidence. Single total transmission peaks are observed. The peak locations are approximately predicted by, but somewhat shifted from, $f_{r, o}^{\text {doc }}=0.136$. The peak locations move only slightly as the periodicity and angle of incidence changes. Figure 4(a) shows the angular dependence $|T(\theta)|$ near local resonances on thick plates. When $f$ is chosen as the frequency that maxizes $|T(f)|$ in Figure 2(a), $|T(\theta)|$ approaches unity over a very wide $\theta$-range - this behavior of course is not observed for $\theta \rightarrow 0$ due to the vanishing coupling of the incident field to the TEM mode. In contrast, when $f$ is chosen away from the local resonance, $|T(\theta)|$ is small, irrespective of $\theta$. In both cases, no peaks in the angular dependence are observed.

\subsection{Surface Waves}

When $\sin \theta>1$, the zeroth-order diffraction mode is an evanescent plane wave and $\cos \theta=-j|\cos \theta|$. Now, the denominators in Eq. (1) have (real) zeros $f_{p}^{\mathrm{sw}}$ with $\sin \theta \equiv \zeta_{p}^{\mathrm{sw}}\left(f_{p}^{\mathrm{sw}}\right)>1[p=o$ and $p=e$ for the odd and even components in Eq. (1), respectively]. The frequencies $f_{o}^{\text {sw }}$ and $f_{e}^{\mathrm{sw}}$ always are obtained for $c q / d<f_{o}^{\mathrm{sw}}<c(2 q+1) /(2 d)$ and $c(2 q+1) /(2 d)<f_{e}^{\text {sw }}<c(q+1) / d$ with integer $q$ $\geq 0$; the corresponding $\zeta_{o}^{\text {sw }}$ and $\zeta_{e}^{\text {sw }}$ increase with $f_{o}^{\text {sw }}$ and $f_{e}^{\text {sw }}$, respectively. On the other hand, for $c(2 q+1) /(2 d)<f_{o}^{\text {sw }}$ $<c(q+1) / d$ and $c q / d<f_{e}^{\mathrm{sw}}<c(2 q+1) /(2 d)$, the frequencies $f_{o}^{\text {sw }}$ and $f_{e}^{\text {sw }}$ exist only above certain cutoff values for which the corresponding $\zeta_{p}^{\mathrm{sw}}=1$. It, therefore, follows that two (odd and even) source-free fields may exist simultaneously. These fields are identified as SWs that are bound to, and propagate along, the perforated plate with the normalized wavenumber $\zeta_{p}^{\mathrm{sw}}$. Unlike the local resonances, the existence of the SWs relies on the interactions between the cavities. It is important to note that for lossless structures, $\left|\zeta_{p}^{\text {sw }}\right|$ can achieve very large values. For instance, in the regime of single zeroth-order diffraction mode they only are limited by the array's periodicities as for $\left|\zeta_{p}^{\mathrm{sw}}\right|>\min \left\{c /\left(2 f_{p}^{\mathrm{sw}} L_{x}\right), c /\left(2 f_{p}^{\mathrm{sw}} L_{y}\right)\right\}$ higher order diffraction modes should be taken into account as in the case of sparser arrays considered next. In the realm of lossy structures, $\left|\zeta_{p}^{\text {sw }}\right|$ may also be limited by loss. The fact that very large SW wavenumbers can be achieved may find use in applications like subwavelength imaging on surface, where the resolution is limited by the SW wavenumber magnitude.

To illustrate the existence of SWs, Figure 2(b) shows the SW dispersion curve, i.e. the dependence $f_{p}^{\mathrm{sw}}\left(\zeta_{p}^{\mathrm{sw}}\right)$, in the regime $\sin \theta$ $=\zeta_{p}^{\mathrm{sw}}>1$ and $c /(2 d)<f_{p}^{\mathrm{sw}}<c / d$ (i.e. $\left.q=1\right)$ for the structure parameters in Figure 2(a). It is found that $\zeta_{e}^{\text {sw }}>\zeta_{o}^{\text {sw }}$ and that $\zeta_{e}^{\text {sw }}$ approaches a large value (up to $c /\left(2 f_{e}^{\mathrm{sw}} L_{x}\right) \approx 1.93$ ) as expected. It should be noted that since for dense arrays the SW exist only for $\sin \theta>1$, they cannot be excited by a propagating incident plane wave and hence cannot lead to transmission enhancement. However, the situation changes for sparse arrays.

\section{WAVE PHENOMENA SUPPORTED BY SPARSE ARRAYS OF COAXIAL HOLES}

This section studies wave phenomena supported by the structure in Figure 1 when the periodicities $L_{x}$ or $L_{y}$ are not small compared to the wavelength. Section 4.1 studies the phenomena of enhanced transmission of obliquely incident plane waves, whereas Section 4.2 considers transmission phenomena under normally incident plane waves.

\subsection{Transmission Phenomena Under Oblique Incidence}

When the periodicities $L_{x}$ or $L_{y}$ are not small compared to the wavelength and/or when the structure is illuminated by a normally incident plane wave, approximate closed-form expression for $T$ can be obtained by keeping additional diffraction modes in the field representation; unfortunately, the resulting expressions are lengthy and somewhat difficult to interpret. We, therefore, elucidate the physical origins of the supported enhanced transmission phenomena for large plate periodicities and normal incidence by extending the above presented framework and by drawing on established phenomenology of enhanced transmission through plates with holes of simply-connected cross-section. The models presented below are supported by numerical examples.

Consider an array with $L_{x}$ and $L_{y}$ that are not small compared to the wavelength (oblique incidence still is assumed). As a result of the interaction between different diffraction modes and the existence of the aforementioned SWs, the structure can support (SW) resonances that are characterized by complex frequencies

$$
f_{n m, p}^{\mathrm{sw}}=f_{r, n m, p}^{\mathrm{sw}}+\delta_{n m, p}^{\mathrm{sw}},
$$

where $f_{r, n m, p}^{\mathrm{sw}}$ are (real) frequencies obtained from the phase matching condition $\left(\left(\sin \theta+2 \pi c n /\left(L_{x} f_{r, n m, p}^{\mathrm{sw}}\right)\right)^{2}+(2 \pi \mathrm{cm} /\right.$ $\left.\left.\left(L_{p} f_{r, n m, p}^{\mathrm{sw}}\right)\right)^{2}\right)^{1 / 2}=\zeta_{p}^{\mathrm{sw}}\left(f_{r, n m, p}^{\mathrm{sw}}\right)$ that approximately predict $f_{n m, p}^{\mathrm{sw}}$, and $\delta_{n m, p}^{\mathrm{sw}}$ are (small) complex-valued displacements. Unlike $f_{p}^{\text {loc }}$, the SW resonance frequencies $f_{n m, p}^{\mathrm{sw}}$ strongly depend on the angle of incidence and the array's periodicities. Due to the SW induced resonances, $|T|$ can have twin transmission peaks as $f$ scans through $\operatorname{Re}\left\{f_{n m, p}^{\mathrm{sw}}\right\}$. Similar, to the local resonances, the peaks exhibit periodic behavior with respect to $d$ and have large magnitudes even for very thick plates. When it comes to the local resonance frequencies $f_{p}^{\text {loc }}$ for sparse arrays, for most angles of incidence they are still given approximately by their dense-array limit. However, when $f_{p}^{\text {loc }}$ approaches $f_{n m, p}^{\mathrm{sw}}$ only combined effects of both resonance types can be observed and the local resonances may be suppressed [18].

To illustrate the existence and properties of the SW resonances, Figure 3(a) depicts the frequency dependence $|T(f)|$ near SW resonances associated with $c /(2 d)<f_{p, 10}^{\mathrm{sw}}<c / d$. As expected, two total transmission peaks are observed. Increasing the plate's thickness leads to a noticeable shift of the lower frequency peak; this is due to the increase of the corresponding $\zeta_{p}^{\text {sw }}$ [see Fig. 2(b)]. Increasing the incident angle leads to a downward shift of both peaks thus verifying the SW dispersive behavior.

\subsection{Transmission Phenomena Under Normal Incidence}

The behavior of $T$ is very different for (near-) normal incidence, i.e. when $\theta \approx 0$. In this regime, the incident plane wave cannot couple to the TEM mode due to the latter's radial symmetry. Instead, the fields couple from one side of the perforated plate to the other mostly through the $\mathrm{TE}_{11}$ evanescent mode. Although local resonances associated with the TEM mode are no longer supported, SW resonances still can be excited. However, the behavior of $f_{n m, p}^{\mathrm{sw}}$ and $|T|$ differs from that discussed above and is similar to that for plates with holes of simply-connected cross-sectional holes [3, 4]. For instance, $f_{n m, e}^{\mathrm{sw}}$ and $f_{n m, o}^{\mathrm{sw}}$ are distinct and $|T|$ exhibits twin transmission peaks for plates of moderate thickness $\left(d<1 /\left(k_{0}\left|\zeta_{11}^{\mathrm{TE}}\right|\right)\right)$, whereas $f_{m n, e}^{\mathrm{sw}} \approx f_{m n, o}^{\mathrm{sw}}$ and $|T|$ has a single reduced transmission peak for thick plates $(d$ $\left.>1 /\left(k_{0}\left|\zeta_{11}^{\mathrm{TE}}\right|\right)\right)$.

Figure 3(b) shows $|T(f)|$ for normal incidence. The obtained behavior is very different than that in Figure 3(a). Just as for simply- 


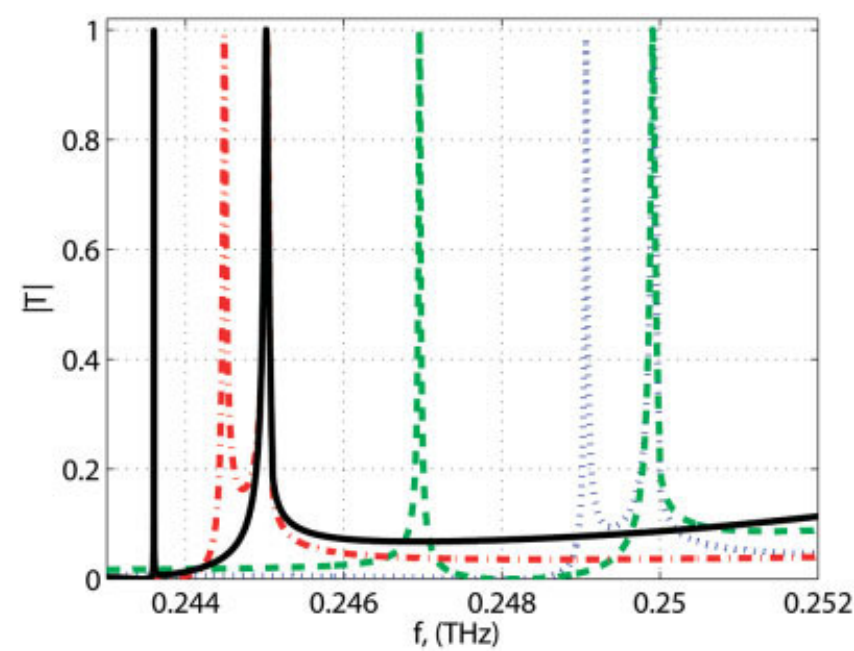

(a)

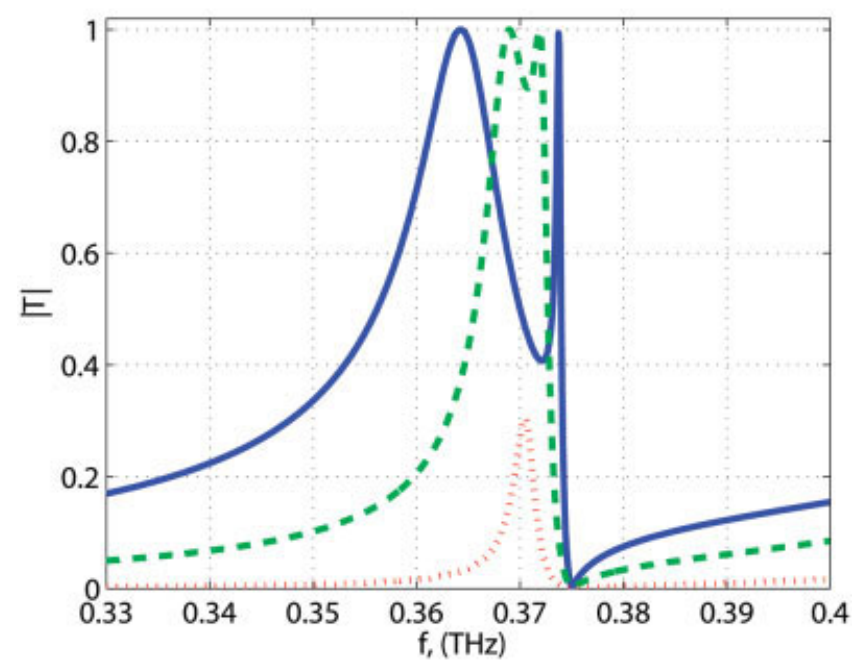

(b)

Figure 3 Transmission coefficient $|T(f)|$. (a) Oblique incidence for $\sin \theta=0.53, d=1.1 \mathrm{~mm}$ (solid line), $\sin \theta=0.5, d=1.1 \mathrm{~mm}$ (dashed line), $\sin \theta=0.5, d=1.05 \mathrm{~mm}$ (dotted line), and $\sin \theta$ $=0.53, d=1.05 \mathrm{~mm}$ (dashed-dotted line); (b) Normal incidence for $d=0.2 \mathrm{~mm}$ (solid line), $d=0.4 \mathrm{~mm}$ (dashed line), and $d$ $=0.9 \mathrm{~mm}$ (dotted line). Other structure parameters are as in Figure 2 and $L_{x}=0.8 \mathrm{~mm}$. [Color figure can be viewed in the online issue, which is available at www.interscience.wiley.com]

connected cross-sectional holes [3, 4], strong double and single transmission peaks with magnitudes that reduce exponentially with plate thickness are obtained for plates of moderate and large thicknesses, respectively. The situation is very different for SW resonances in Figure 4(b) . Here, two resonant total transmission peaks are obtained that qualitatively behave similarly to those in Figure 3(a).

\section{SUMMARY}

PEC plates perforated by periodic arrays of subwavelength coaxial through-holes permit enhanced transmission phenomena and SW guidance phenomena. The enhanced transmission phenomena are supported even in the regime where all higher order modes in the through-holes are cut-off. In contrast to past studies [10-12], the enhancement is attributed to the coupling of free-space fields not only to the $\mathrm{TE}_{11}$ modal fields but also to TEM ones and is associated with two mechanisms. One is a local coaxial cavity resonance of TEM modal fields supported by individual cavities. The other is the coupling between diffraction modes and SWs that arise due to the cavity interactions. The SWs can have large propagation wavenumbers, which can be controlled the structural parameters, e.g. the plate thickness. The transmission coefficient behavior is different for oblique and normal incidence. For the former and latter case this behavior is similar to that obtained for infinite slits [17-19] and simply-connected cross-sectional holes [3, 4], respectively.

The study in this letter compliment other studies on the scattering from holes of coaxial cross-section by considering the regime of sparse arrays and deeply subwavelength hole sizes and identifying/elucidating the effects introduced by the presence of TEM modes and SWs. The presented PEC model is

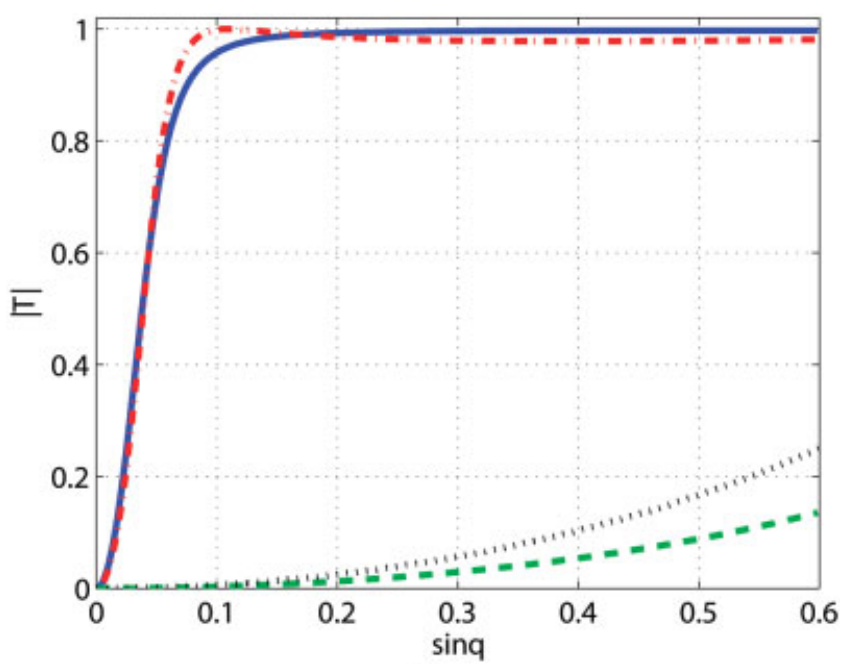

(a)

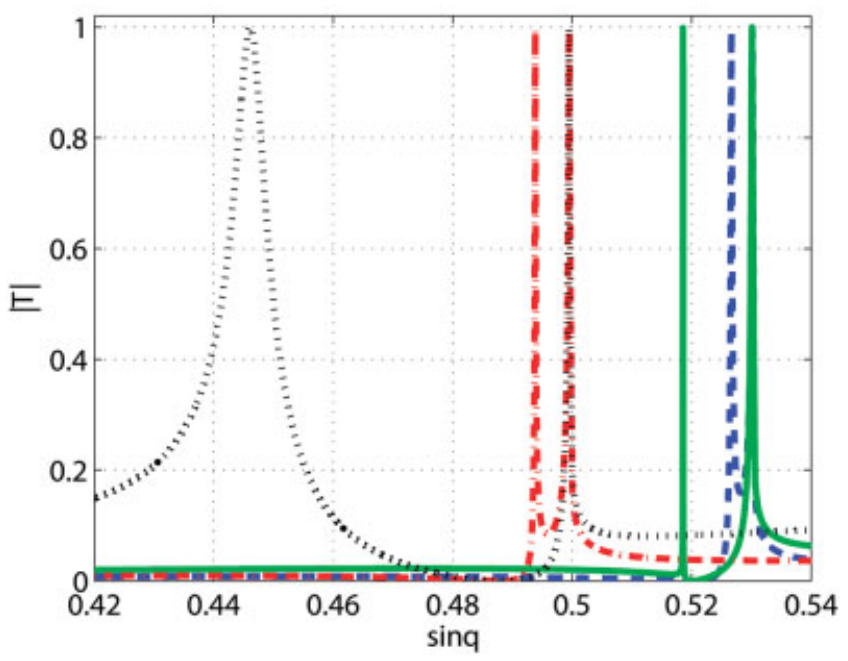

(b)

Figure 4 Transmission coefficient $|T(\theta)|$. (a) Regime of local resonances for $d=1.1 \mathrm{~mm}$ and for $f=0.12785 \mathrm{THz}, L_{x}=0.31 \mathrm{~mm}$ (solid line), $f=0.125 \mathrm{THz}, L_{x}=0.31 \mathrm{~mm}$ (dashed line), $f=0.12651 \mathrm{THz}, L_{x}$ $=0.8 \mathrm{~mm}$ (dashed dotted line), $f=0.126 \mathrm{THz}, L_{x}=0.8 \mathrm{~mm}$ (dotted line); (b) SW resonances for $L_{x}=0.8 \mathrm{~mm}$ and for $f=0.245 \mathrm{THz}, d$ $=1.1 \mathrm{~mm}$ (solid line), $f=0.245 \mathrm{THz}, d=1.05 \mathrm{~mm}$ (dashed line), $f$ $=0.25 \mathrm{THz}, d=1.1 \mathrm{~mm}$ (dotted line), and $f=0.25 \mathrm{THz}, d$ $=1.05 \mathrm{~mm}$ (dashed dotted line). Other structure parameters are as in Figure 2. [Color figure can be viewed in the online issue, which is available at www.interscience.wiley.com] 
adequate in the microwave and terahertz regimes and provides insights into related wave phenomena in the optical regime. It is noted that in the optical regime near the metal plasma frequency, additional wave phenomena that further complicate the structure's angular or frequency response may occur; these include strong excitation of higher-order cavity modes and surface plasmon polariton coupling [10-12]. The presented structure and its derivatives can be used to construct periodic grating filters, near- and far-field probes, antennas, and high impedance surfaces.

\section{REFERENCES}

1. T.W. Ebbesen, H.J. Lezec, H.F. Ghaemi, T. Thio, and P.A. Wolff, Extraordinary optical transmission through sub-wavelength hole arrays, Nature 391 (1998), 667-669.

2. L. Martín-Moreno, F.J. García-Vidal, H.J. Lezec, K.M. Pellerin, T. Thio, J.B. Pendry, and T.W. Ebbesen, Theory of extraordinary optical transmission through subwavelength hole array, Phys Rev Lett 86 (2001), 1114-1117.

3. V. Lomakin, N.W. Chen, S.Q. Li, and E. Michielssen, Enhanced transmission through two-period arrays of sub-wavelength holes, IEEE Microw Wireless Compon Lett 14 (2004), 355-357.

4. V. Lomakin and E. Michielssen, Enhanced transmission through metallic plates perforated by arrays of subwavelength holes and sandwiched between dielectric slabs, Phys Rev B (Condens Matter Mater Phys) 71 (2005), 235117.1-235117.10.

5. J.G. Rivas, C. Schotsch, P.H. Bolivar, and H. Kurz, Enhanced transmission of THz radiation through subwavelength holes, Phys Rev B (Condens Matter Mater Phys) 68 (2003), 201306.1201306.4.

6. M. Beruete, M. Sorolla, I. Campillo, J.S. Dolado, L. Martin-Moreno, J. Bravo-Abad, and F.J. Garcia-Vidal, Enhanced millimeter wave transmission through quasioptical subwavelength perforated plates, IEEE Trans Antennas Propag 53 (2005), 1897-1903.

7. A. Roberts and R.C. McPhedran, Bandpass grids with annular apertures, IEEE Trans Antennas Propag 36 (1988), 607-611.

8. F.I. Baida and D. Van Labeke, Light transmission by subwavelength annular aperture arrays in metallic films, Opt Commun 209 (2002), 17-22.

9. F.I. Baida and D. Van Labeke, Three-dimensional structures for enhanced transmission through a metallic film: Annular aperture arrays, Phys Rev B 67 (2003), 155314.1-155314.7.

10. F.I. Baida, D. Van Labeke, G. Granet, A. Moreau, and A. Belkhir, Origin of the super-enhanced light transmission through a 2-D metallic annular aperture array: A study of photonic bands, Appl Phys B (Lasers Opt) 79 (2004), 1-8.

11. J. Salvi, M. Roussey, F.I. Baida, M.-P. Bernal, A. Mussot, T. Sylvestre, H. Maillotte, D. Van Labeke, A. Perentes, I. Utke, C. Sandu, P. Hoffmann, and B. Dwir, Annular aperture arrays: Study in the visible region of the electromagnetic spectrum, Opt Lett 30 (2005), 1611-1613

12. W. Fan, S. Zhang, B. Minhas, K.J. Malloy, and S.R.J. Brueck, Enhanced infrared transmission through subwavelength coaxial metallic arrays, Phys Rev Lett 94 (2005), 033902.1-033902.4.

13. B. Hecht, B. Sick, U.P. Wild, V. Deckert, R. Zenobi, O.J.F. Martin, and D.W. Pohl, Scanning near-field optical microscopy with aperture probes: Fundamentals and applications, J Chem Phys 112 (2000), 7761-7774.

14. K.M. Pellerin, H.J. Lezec, T.W. Ebbesen, R.A. Linke, and T. Thio, Giant optical transmission of subwavelength apertures for NSOM and data storage, In: Proceedings of IEEE Conference, 2001, pp 293-298.

15. P.F. Goldsmith, Quasioptical sistems-Gaussian beam, quasioptical propagation, and applications, IEEE Press, Piscataway, NJ, 1998.

16. T. Thio, H.J. Lezec, T.W. Ebbesen, K.M. Pellerin, G. D. Lewen, A. Nahata, and R.A. Linke, Giant optical transmission of sub-wavelength apertures: Physics and applications, Nanotechnology 13 (2002), 429-432.
17. J.R. Andrewartha, J.R. Fox, and I.J. Wilson, Resonance anomalies in the lamellar grating, Opt Acta 26 (1979), 69-89.

18. Q. Cao and P. Lalanne, Negative role of surface plasmons in the transmission of metallic gratings with very narrow slits, Phys Rev Lett 88 (2002), 057403.

19. F.J. Garcia-Vidal and L. Martin-Moreno, Transmission and focusing of light in one-dimensional periodically nanostructured metals, Phys Rev B 66 (2002), 155412.

20. C.-C. Chen, Transmission of microwave through perforated flat plates of finite thickness, IEEE Trans Microwave Theory Tech MTT21 (1973), 1-6

(C) 2007 Wiley Periodicals, Inc.

\section{A SIMPLE SPICE MODEL FOR TRAVELING WAVE SEMICONDUCTOR LASER AMPLIFIER}

\author{
Abhirup Das Barman, ${ }^{1}$ Ipsita Sengupta, ${ }^{2}$ and P. K. Basu ${ }^{1}$ \\ ${ }^{1}$ Institute of Radio Physics and Electronics, University of Calcutta, 92 \\ Acharya Prafulla Chandra Road, Kolkata 700 009, India \\ ${ }^{2}$ ECE Department of Hooghly Engineering and Technology, Pipulpati, \\ Hooghly, West Bengal 712103, India
}

Received 7 December 2006

\begin{abstract}
An equivalent lumped element electric circuit model for traveling wave semiconductor laser amplifier has been developed using the rate equation for carriers. SPICE simulation of the circuits gives results in close agreement with the experimental data and values from sophisticated analyses. The modulation bandwidth and transient response were examined by including parasitic elements in the intrinsic model. () 2007 Wiley Periodicals, Inc. Microwave Opt Technol Lett 49: 1558-1561, 2007; Published online in Wiley InterScience (www. interscience.wiley.com). DOI 10.1002/mop.22483
\end{abstract}

Key words: traveling wave semiconductor laser optical amplifier; lumped circuit model; electrical parasitics

\section{INTRODUCTION}

Semiconductor laser optical amplifiers (SLAs) are increasingly being used as amplifiers, routers, wavelength converters, cross connects, and logic gates in present day fiber optic communication and networking systems. A number of theoretical models: both analytical and numerical, have been proposed in the literature [1-8] for predicting the operational characteristics of the SLAs. However, the numerical models, like transfer matrix method (TMM) [9] or transmission line matrix method [10] work better for a few specific analyses, either steady state or transient behavior.

In the present work, we develop a simple lumped circuit model for SLAs which can predict both steady state and transient response by using a SPICE simulator. Similar equivalent circuits have been developed earlier for laser diodes [11], quantum well lasers [12], photodetectors and photoreceivers [13], and recently for quantum cascade lasers [14]. Our circuit has been developed by using rate equations for carrier densities in a traveling wave SLA (TW-SLA). The results of our simulation agree closely with experimental data as well as with values obtained from TMM analysis. Inclusion of parasitic elements, like package capacitance, etc, in the intrinsic circuit model provides information on modulation bandwidth and transient response. 